\title{
Lusioersily
}

\section{Analysis of excitation processes and electron temperature changes from spectral data in a dc micro plasma discharge}

Mariotti, D., Maguire, PD., Mahony, CMO., \& McLaughlin, JAD. (2004). Analysis of excitation processes and electron temperature changes from spectral data in a dc micro plasma discharge. Plasma Sources Science and Technology, 13(4), 576-581. https://doi.org/10.1088/0963-0252/13/4/004

Link to publication record in Ulster University Research Portal

\section{Published in:}

Plasma Sources Science and Technology

Publication Status:

Published (in print/issue): 01/01/2004

DOI:

10.1088/0963-0252/13/4/004

\section{Document Version}

Publisher's PDF, also known as Version of record

\section{General rights}

Copyright for the publications made accessible via Ulster University's Research Portal is retained by the author(s) and / or other copyright owners and it is a condition of accessing these publications that users recognise and abide by the legal requirements associated with these rights.

\section{Take down policy}

The Research Portal is Ulster University's institutional repository that provides access to Ulster's research outputs. Every effort has been made to ensure that content in the Research Portal does not infringe any person's rights, or applicable UK laws. If you discover content in the Research Portal that you believe breaches copyright or violates any law, please contact pure-support@ulster.ac.uk. 


\title{
Analysis of excitation processes and electron temperature changes from spectral data in a de micro plasma discharge
}

\author{
Davide Mariotti, Paul Maguire, Charles M O Mahony and \\ James McLaughlin
}

NIBEC, University of Ulster at Jordanstown, Newtownabbey, BT37 0QB, UK

Received 12 May 2004

Published 9 September 2004

Online at stacks.iop.org/PSST/13/576

doi:10.1088/0963-0252/13/4/004

\begin{abstract}
A micro scale dc plasma discharge was studied to determine the potentialities for lab-on-the-chip applications. Different working conditions for the micro plasma discharge were considered: the pressure was varied between 2.7 and $5.3 \mathrm{kPa}$ and the applied dc voltage was between 400 and $540 \mathrm{~V}$, generating a discharge current in the range of $0.02-0.09 \mathrm{~mA}$. The electrode distance was maintained at $0.025 \mathrm{~cm}$ and argon was used as the gas for the formation of plasma discharges. The number densities of excited states were determined by spectral emission data $(400-1000 \mathrm{~nm})$ and then were calculated by introducing a few assumptions: comparison of experimental and calculated number densities allowed an analysis of a volume averaged electron energy distribution function. Also, it was possible to estimate an effective electron temperature for different conditions of pressure and applied voltage.
\end{abstract}

\section{Introduction}

In recent years interest in lab-on-the-chip applications has grown due to technological advances in device miniaturization; plasmas have therefore been studied for integration in such miniature systems [1-6]. A dc driven micro plasma device (MPD) has been developed here for use in gas analysis and artificial olfaction applications by mean of plasma emission spectroscopy [1,7-9]. In this paper, some of the physical properties of the micro scale plasma generated by the MPD have been studied in terms of the electron energy distribution and by means of an effective electron temperature, which is explained in the following sections.

Plasma spectroscopy relies on the excitation of plasma species and subsequent de-excitation by radiative decay. The nature and the frequency of excitation processes are therefore fundamental in the production of spectral data [10]; plasma excitation processes determine spectral line availability and strongly affect line intensity. One of the main excitation processes occurs by electron collision and therefore it is useful to have information about the electron energy distribution, particularly over the excitation energy range. Analysis of electron behaviour can contribute to an understanding of spectral emission and help determine the potential of plasma spectroscopy for gas analysis applications.

In this paper, the electron energy distribution function (EEDF) behaviour has been evaluated from argon spectral emission. Experimental spectral lines have been used to calculate excited levels number densities, which provide a distribution over energy that is closely related to the electron energy distribution. Using a limited set of assumptions, the same distribution can be numerically calculated, compared with the experimental results, and an estimate of the effective electron temperature obtained. The comparison can also reveal changes in electron temperature by varying the pressure and applied voltage.

\section{The MPD}

A custom MPD was manufactured and complemented with equipment for measuring electrical parameters and spectral emission [1]. The MPD itself is a small glass vessel sealed 


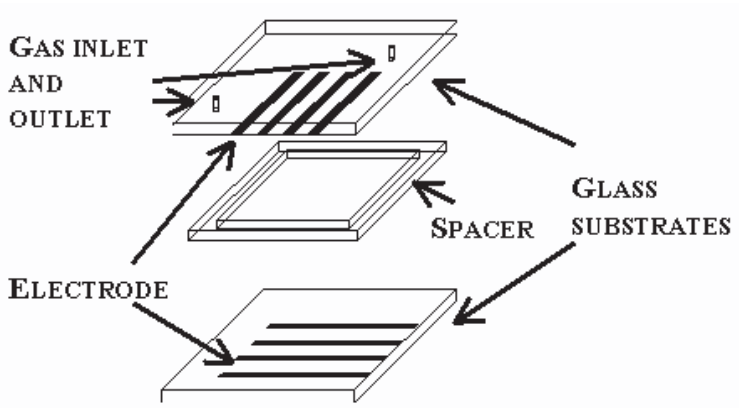

Figure 1. MPD before being assembled: the glass substrates are on top and bottom of the drawing and the PTFE film spacer is in between. Both glass substrates have four metallic electrodes. The top glass substrate also has a gas inlet and outlet.

with epoxy with two holes in the glass to allow a gas inlet and outlet. The experimental configuration of the MPD is best seen in figure 1, which depicts the device before assembly. Two glass substrates with four patterned electrodes each are placed one on top of the other and are kept separate by a square frame of polytetrafluoroethylene (PTFE) film spacer. The three components are brought together and clamped before being sealed with epoxy. This matrix arrangement forms 16 pixels where electrodes cross each other and with a gap distance determined by the choice of spacer thickness. The glass substrates are obtained from commercial k-glass (Pilkington), which has, on one side, a transparent conducting fluorine-doped tin oxide $\left(\mathrm{SnO}_{2}: \mathrm{F}\right)$ layer (about $250 \mathrm{~nm}$ thick). Electrodes were obtained by etching the tin oxide according to the desired pattern (as shown in figure 1). Despite the complexity of the MPD as a whole, each single pixel presents a simple parallel plate configuration. The geometry and the plasma volume are therefore defined by the electrode width $(0.3 \mathrm{~cm})$ and the spacer thickness $(0.025 \mathrm{~cm})$, i.e. $0.3 \mathrm{~cm} \times$ $0.3 \mathrm{~cm} \times 0.025 \mathrm{~cm}$.

This confined plasma device differs significantly from other plasma devices built using a coplanar structure [11]. The latter typically have a much greater inter-electrode spacing, a few millimetres, compared with $0.025 \mathrm{~cm}$ here, and the plasma region is less easily defined. Therefore coplanar devices are likely to require higher energy electrons to initiate and sustain the plasma and exhibit less plasma and current density uniformity.

Each pixel can be individually activated by selecting an electrode pair, and consequent emission from a single pixel can be acquired. A lab-built power supply and measurement system provided a dc voltage ranging from 0 to $600 \mathrm{~V}$ in series with a variable limiting resistor $(0.5-10 \mathrm{M} \Omega)$. The voltage could be applied individually to each of the 16 pixels by selection of the appropriate electrode pair. Electrical parameters could be measured, and an analysis of some of the electrical characteristics of the device has been reported [12]. The arrangement of the spectra acquisition system can be seen in figure 2, which also shows a cross-section of the MPD and the fibre holder used to secure the optical fibre in position. An optical fibre captures the spectral emission from the anode side of the plasma. The anode was preferred to the cathode as the optical transparency of the latter may degrade due to high energy ion bombardment [9]. The light is then transferred to a

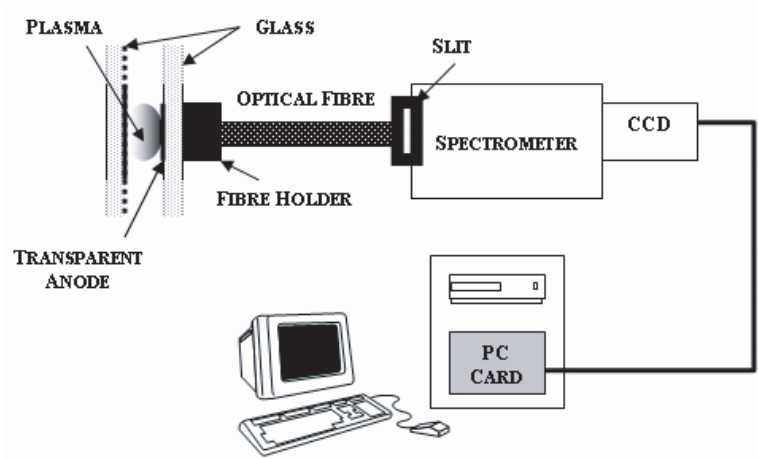

Figure 2. Schematic diagram depicting the spectra acquisition system configuration. The emission is gathered from the anode side of the glass by an optical fibre. A holder keeps the fibre in place. The spectrophotometer and PC interface allow reading of the spectral data.

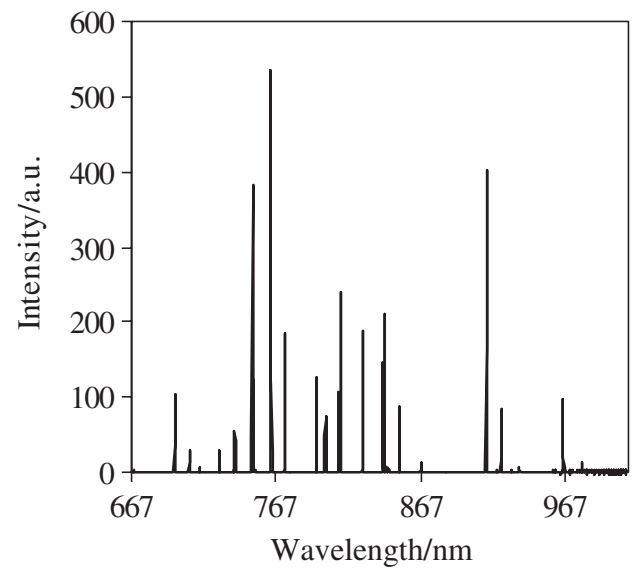

Figure 3. Argon spectra at $4 \mathrm{kPa}$ and $0.092 \mathrm{~mA}$ discharge current.

spectrophotometer for acquisition (figure 2). The wavelengths were calibrated with a second order polynomial equation using three prominent argon lines. Intensity relative calibration to make up for instrumental light attenuation has been obtained according to a certified light source.

It is important to note that the spectral emission, collected from the transparent anode, represents the sum of the emission along the discharges axis within the optical fibre acceptance angle, i.e. end-on observation [13], since the plasma is assumed to be optically thin. This also means that spectral information is generated from plasma regions that are in principle at different conditions (different electric field, electron energy, etc). As a consequence, the spectral line intensities may contain an added degree of complexity and can be considered averaged over the whole plasma. A typical spectral emission at $4 \mathrm{kPa}$ and $0.092 \mathrm{~mA}$ discharge current is shown in figure 3 . No emission lines could be detected outside the ranges reported in figure 3 . The instrument acquisition wavelength range was from 400 to $1000 \mathrm{~nm}$.

\section{Experimental number densities of excited states}

There are a number of processes occurring within the plasma volume that determine the density of gas particles in each 
Table 1. Configuration term, total electronic angular momentum $(J)$ and corresponding energy utilized for calculation of number densities. The spectral lines listed are those relative to transition from the same upper energy state, and the last column on the right indicates the availability of cross-section data for this analysis [14-16].

\begin{tabular}{|c|c|c|c|c|}
\hline $\begin{array}{l}\text { Configuration } \\
\text { term }\end{array}$ & $J$ & $\begin{array}{l}\text { Energy } \\
\text { threshold } \\
(\mathrm{eV})\end{array}$ & $\begin{array}{l}\text { Spectral } \\
\text { lines }(\mathrm{nm})\end{array}$ & $\begin{array}{l}\text { Cross-section } \\
\text { data availability }\end{array}$ \\
\hline $6 s^{2}[3 / 2]^{\circ}$ & 2 & 14.83881 & 703.2213 & No \\
\hline $4 d^{2}[1 / 2]^{\circ}$ & 1 & 14.71090 & 687.3160 & Yes \\
\hline $5 s^{2}[1 / 2]^{\circ}$ & 1 & 14.25509 & 919.7098 & No \\
\hline $4 p^{2}[1 / 2]^{\circ}$ & 0 & 13.47989 & 750.5917 & Yes \\
\hline $4 p^{2}[1 / 2]^{\circ}$ & 1 & 13.32786 & $\begin{array}{l}696.7325 \\
727.4918 \\
826.6774\end{array}$ & Yes \\
\hline $4 p^{2}[3 / 2]^{\circ}$ & 2 & 13.30223 & $\begin{array}{l}706.9141 \\
738.5994 \\
841.0501\end{array}$ & Yes \\
\hline $4 p^{2}[3 / 2]^{\circ}$ & 1 & 13.28264 & $\begin{array}{l}714.8991 \\
795.0358 \\
852.3773\end{array}$ & No \\
\hline $4 p^{2}[1 / 2]^{\circ}$ & 0 & 13.27304 & 751.6702 & No \\
\hline $4 p^{2}[3 / 2]^{\circ}$ & 2 & 13.17178 & $\begin{array}{l}763.7178 \\
800.8335 \\
922.7009\end{array}$ & Yes \\
\hline $4 p^{2}[3 / 2]^{\circ}$ & 1 & 13.15314 & $\begin{array}{l}810.5929 \\
867.0348 \\
935.6807\end{array}$ & Yes \\
\hline $4 p^{2}[5 / 2]^{\circ}$ & 2 & 13.09487 & $\begin{array}{l}801.6983 \\
842.6965 \\
978.7200\end{array}$ & Yes \\
\hline $4 p^{2}[5 / 2]^{\circ}$ & 3 & 13.07572 & 811.7499 & Yes \\
\hline $4 p^{2}[1 / 2]^{\circ}$ & 1 & 12.90701 & $\begin{array}{l}912.5480 \\
966.0457\end{array}$ & Yes \\
\hline
\end{tabular}

excited energy state. The density of excited argon atoms is proportional to the spectral emission intensity and the following relationship can be written:

$$
n_{k} \propto \frac{I_{k i} \lambda_{k i}}{A_{k i}}
$$

where $I_{k i}$ is the line intensity of the transition from energy level $k$ to $i, A_{k i}$ is the transition probability, $n_{k}$ is the particle number density at the excited state $k$ and $\lambda_{k i}$ is the wavelength of the spectral line. The relation holds for optically thin plasmas and when spontaneous radiative decay is the only de-excitation process. Here both conditions can be assumed satisfied [14].

Using transition probabilities from [14-16] (see table 1) relative excited state number densities for a number of spectral lines were calculated. The number densities for each argon energy state are plotted in figure 4 as a function of energy and for a representative set of discharge conditions $(450 \mathrm{~V}$, $0.064 \mathrm{~mA}, 4 \mathrm{kPa})$. The densities have been normalized to the value of the number density at an energy level of $14.7109 \mathrm{eV}$ $\left(4 \mathrm{~d}^{2}[1 / 2]^{\circ} J=1\right)$, which has been set to 1 . The number densities at the same energy level and calculated from different line intensities have been averaged and the error bars of figure 4 represent the corresponding standard deviations. The accuracy of the results is often limited by the uncertainties of the transition probabilities [17-19]. Most of the energy levels refer to $4 \mathrm{p}$ levels as listed in table 1 . The only exceptions are the three rightmost data points.

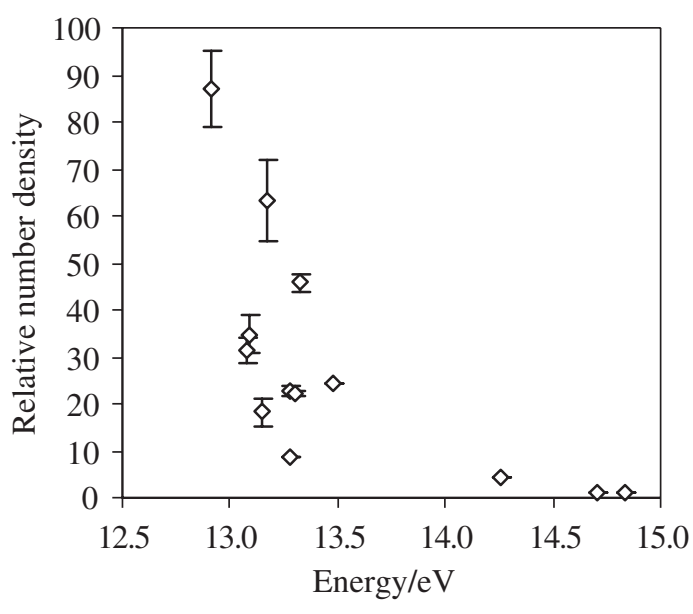

Figure 4. Number density as calculated from emission measurements. The applied voltage was set to $450 \mathrm{~V}$ for a discharge current of $0.064 \mathrm{~mA}$. The pressure was set to $4 \mathrm{kPa}$. The density at $14.7109 \mathrm{eV}$ is set to 1 and all the other values are presented relative to this. Error bars refer to uncertainty due to measurement.

In general, the relative number density decreases with increasing energy and this can be attributed mainly to a reduced electron density at higher energies, assuming only electronatom collisions are considered. For other possible excitation processes, any particles undergoing inelastic collisions with argon atoms also have a decreasing density with increasing energy profile and generally contribute to excitation only with the tail of their respective energy distribution functions. The scattering of the data in figure 4 can be attributed to different electron collision excitation cross-sections from the ground state and also from metastable states. Population of metastable states $\left(4 \mathrm{~s}^{2}[3 / 2]^{\circ} J=2\right.$ and $\left.4 \mathrm{~s}^{2}[1 / 2]^{\circ} J=0\right)$ may contribute significantly to both the population exponential increase with decreasing energy and to the scattering of the data points in the left side of the graph. These two metastable states have a relatively low threshold energy (4s levels) and have shown preferential step-wise excitation paths [14]. Lower energy excited levels can also be populated by cascading from higher levels, although it seems to be less important as (figure 4 ) the higher energy levels have very low number densities [20]. This is also confirmed by the fact that no other lines from 3d, 5p, 6s, 6p and 7s levels were observed, some of which are often measured in argon plasmas (e.g. 427.2169, 592.8812 and $731.1716 \mathrm{~nm}$ ) [13].

Number density calculations from line intensities have been performed for a range of discharge conditions where the pressure was varied from 2.7 to $5.3 \mathrm{kPa}$ and the applied voltage from 400 to $540 \mathrm{~V}$, corresponding to a discharge current between about 0.02 and $0.09 \mathrm{~mA}$. All the data obtained at different experimental conditions have exhibited very similar behaviour, and the same considerations apply.

\section{Calculated number densities and evaluation of electron temperature}

The micro plasma studied here can be classified as a lowdensity plasma in which the electron density and ion density are low compared with the total gas density. This property has a very important consequence, which sets limits to the 
physical processes that can take place. Because of the low charge density, excited gas particles will experience collisions at a rate that is very low compared with the de-excitation rate by radiative decay [21]. This means that local thermodynamic equilibrium (LTE) does not apply and Boltzmann distributions should not in general be assumed [22]. Also it is possible to neglect de-excitation processes due to collisions, as particles in excited states would not live sufficiently long. For the same reason step-wise excitation and excitation due to energetic particles are negligible, with the exception of those that involve metastable states. It can be said that the corona model is almost satisfied, for which the excitation from ground level due to electron collisions is fully balanced by radiative decay [23, 24]. However, excitation by ions/fast particles and the influence of metastable states are not included in the corona model [25]. The main collision processes that could contribute to the population of excited levels are therefore summarized below:

(a) $\mathrm{e}^{-}+\mathrm{Ar} \rightarrow \mathrm{Ar}^{*}+\mathrm{e}^{-}$

(b) $\mathrm{Ar}^{+}+\mathrm{Ar} \rightarrow \mathrm{Ar}^{+}+\mathrm{Ar}^{*}$

(c) $\mathrm{Ar}^{\text {fast }}+\mathrm{Ar} \rightarrow \mathrm{Ar}+\mathrm{Ar}^{*}$

(d) $\mathrm{X}+\mathrm{Ar}^{\mathrm{m}} \rightarrow \mathrm{X}+\mathrm{Ar}^{*}$,

where $\mathrm{e}^{-}$represents electrons and Ar represents argon atoms, while $\mathrm{Ar}^{+}, \mathrm{Ar}^{*}, \mathrm{Ar}^{\mathrm{m}}$ and $\mathrm{Ar}^{\text {fast }}$ refer to argon ions, excited atoms, metastables and fast atoms, respectively; $\mathrm{X}$ is any of the particles involved in collisions with metastables. Assuming that the only significant excitation process occurring is direct excitation by electrons, process (a), then the excited level number densities can be determined from excitation crosssections using the corona model if an estimate for the EEDF is introduced. The proportionality relation among number density, EEDF and cross-section for each energy state $k$ is given by the following equation:

$$
n_{k} \propto \frac{\int_{0}^{\infty} \sigma_{0 k}(\varepsilon) \sqrt{2 \varepsilon / m_{\mathrm{e}}} g(\varepsilon) \mathrm{d} \varepsilon}{\sum_{i} A_{k i}},
$$

where $\sigma_{0 k}$ is the excitation cross-section by an electron collision from the ground state to $k$ level, $g$ is the normalized EEDF and $\varepsilon$ is the energy [26]. Equation (2) applies under steady state conditions and for uniform plasma conditions. The use of equation (2) is an additional approximation to the corona assumptions as plasma conditions are not uniform over the volume of the micro plasma discharge.

Cross-section data have been taken from unpublished tabulated data, which have been compared with experimental data and other calculated cross-sections [15]. These data report excitation cross-sections for electron-argon atoms collisions to several $4 p$ levels (red lines) and to several other higher energy states; specifically, the available data are as indicated in table 1 .

The choice of the EEDF is a very important one, and given the available information it is not possible to assume a particular EEDF with a high degree of confidence. The literature reports different EEDFs for different plasma conditions and configurations, although there are no specific results for the set of parameters/configuration used within this work [11,23,27,28]. A bi-Maxwellian EEDF or the presence of a high-energy tail is possible and could be taken into account. Nevertheless, from an emission point of view, energetic electrons, from a high-energy tail, do not contribute substantially to excitation processes. Excitation cross-sections of electron-argon atom collisions decrease quite rapidly with increasing energy beyond $80-100 \mathrm{eV}$. The same considerations apply for the ionization processes, as the corresponding cross-sections also fall quite rapidly at high energy. Compared with other coplanar devices, the elastic collision losses here are expected to be much less significant due to the greatly reduced path length and thus the plasma can be sustained at much lower electron energies. In this situation, therefore, the use of a Maxwell-Boltzmann distribution for the calculation is reasonable. The suitability of this approach will be discussed more fully later when comparing the number densities extracted from the spectral emission to calculated values. The expression for the normalized Maxwell-Boltzmann distribution, $g$, is

$$
g(\varepsilon)=\sqrt{\frac{4 \varepsilon}{\pi\left(k T_{\mathrm{e}}\right)^{3}}} \exp \left(\frac{-\varepsilon}{k T_{\mathrm{e}}}\right),
$$

where $k$ is the Boltzmann constant and $T_{\mathrm{e}}$ is the electron temperature.

The integral in equation (2) can be calculated numerically as the cross-section data are tabulated for discrete energy values only and the formula utilized for the calculations is given in (4),

$\int_{0}^{\infty} \sigma_{k}(\varepsilon) \sqrt{\frac{2 \varepsilon}{m_{\mathrm{e}}}} g(\varepsilon) \mathrm{d} \varepsilon \approx \sum_{i=1}^{N-1} \sigma_{k}\left(\varepsilon_{i}\right) \sqrt{\frac{2 \varepsilon_{i}}{m_{\mathrm{e}}}} g\left(\varepsilon_{i}\right)\left(\varepsilon_{i+1}-\varepsilon_{i}\right)$

where $N$ is the number of tabulated cross-section values for the energy level $k, \varepsilon_{i}$ is the $i$ th tabulated energy value, $\sigma_{k}\left(\varepsilon_{i}\right)$ is the tabulated cross-section value for the energy $\varepsilon_{i}$ and $g\left(\varepsilon_{i}\right)$ is calculated from equation (3).

Using equations (3) and (4) in equation (2), the relative number densities can be calculated under the assumptions introduced previously, i.e. only process (a) contributes to excitation processes. These relative number densities also depend on the electron temperature, which is unknown. In order to compare number densities obtained with equation (2) and those determined from spectral emission, equation (1), the density of the $4 \mathrm{~d}^{2}[1 / 2]^{\circ}(J=1)$ level has been set to the same value on the assumption that the higher energy levels are relatively immune to cascading and to the excitation processes (b)-(d), which are not incorporated in the corona model $[13,29]$. Effectively, this means keeping the $4 \mathrm{~d}^{2}[1 / 2]^{\circ}(J=1)$ density value equal to 1 as experimental number densities were normalized with respect to this energy level, being the highest energy state for which cross-section data were available.

At this stage, modification of the electron temperature provides an opportunity to fit calculated number densities to the experimental densities obtained in the previous section. The fitting procedure can be performed by minimizing the error for a specific energy level. Figure 5 shows the different values of electron temperature obtained by minimizing the error of each of the energy levels one at a time but with the same settings of pressure and voltage ( $4 \mathrm{kPa}$ pressure, $450 \mathrm{~V}$ applied voltage and $0.064 \mathrm{~mA}$ current). The distribution of electron temperature in figure 5 has a relative standard deviation of $11.21 \%$. Under different conditions of pressure and applied voltage the electron temperature standard deviation varied between $9.5 \%$ and $12 \%$, equivalent to $\pm 260 \mathrm{~K}$. 


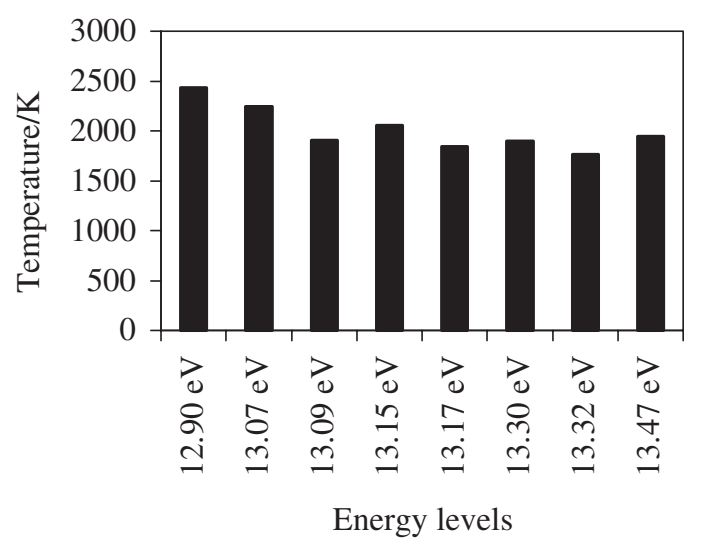

Figure 5. Electron temperature values obtained by fitting calculated number densities to experimental results; each temperature value represents the fitting procedure by minimizing the error of a specific energy level. The plasma conditions were $4 \mathrm{kPa}$ pressure, $450 \mathrm{~V}$ applied voltage and $0.064 \mathrm{~mA}$ current.

These fluctuations may be due to the contribution of particles other than electrons. In fact, the calculated densities have been obtained by assuming that the only populating process of the excited levels was excitation by electron collision. Figure 5 could indicate that other populating processes take place, which by assumption are less influential at higher energy levels. Excitation collisions by ions, fast argon atoms and metastables, as in processes (a)-(d), could all participate in excitation of argon atoms. Certainly, discrepancies between calculated and measured population densities can be attributed to the particular EEDF used for the calculations; the actual distribution is likely to be different from a Maxwell-Boltzmann distribution. Moreover, one uniform EEDF over the whole plasma discharge is not expected, especially for high voltages and high currents, when the electric field is not uniform and presents strong distortion along the discharge axis.

Nevertheless, it seems that the major contribution to excitation remains that of process (a) and that the electron temperature standard deviation can be considered well within calculation approximations and experimental errors, also in consideration of the accuracy of transition probabilities. Although these results cannot reveal conclusive information about the true EEDF and its variation over the discharge volume, it is clear that a uniform Maxwell-Boltzmann distribution satisfactorily models the overall plasma operation with respect to the excitation processes. This also suggests that by averaging the derived temperature values, at each pressure and applied voltage, we can obtain an approximate or effective electron temperature for that condition. While these absolute values of effective electron temperature may not be accurate, considering all the assumptions involved, their relative variation can provide useful insight as plasma parameters are changed.

\section{Effect of pressure and current on electron temperature}

As has been shown previously, an effective electron temperature can be estimated by fitting calculated number

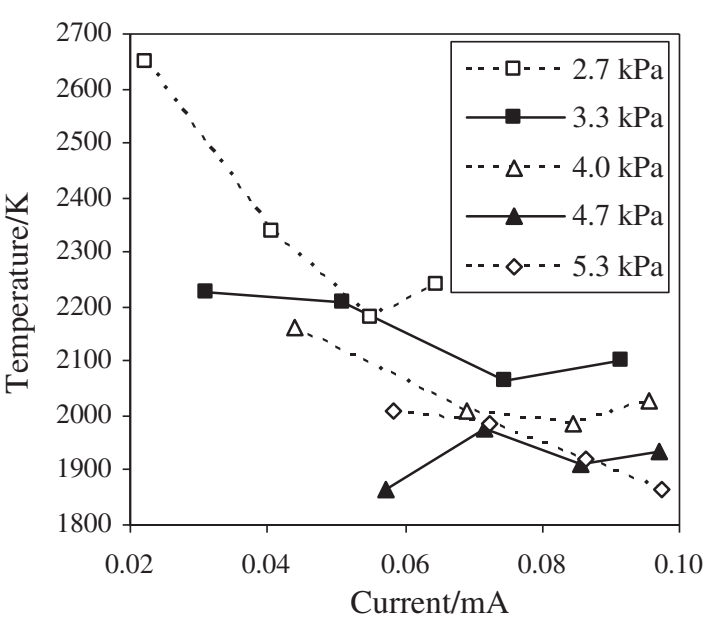

Figure 6. Electron temperature versus discharge current at different pressure values. Temperatures have been obtained by fitting calculated number densities to experimental data.

density with experimental data. The electron temperature calculated in this way would correspond to the actual electron temperature if thermodynamic equilibrium could be assumed and if all the assumptions of the corona model are satisfied, which is not the case here. As has been discussed earlier, the electron temperature calculated earlier is related to the central value of the actual electron distribution and it can be used to observe changes in the electron distribution by varying the plasma discharge conditions, such as the pressure and applied voltage $[23,30]$. The averaged temperature values, obtained as described in the previous section, are shown against current, corresponding to changes in applied voltage, for several pressure values (figure 6). The temperature tends to drop with increasing pressure, as the energy exchange between the electric field and electrons is reduced. The temperature seems to drop with increasing current as well, before levelling off at higher currents. This plasma device operates at a point on the $I-V$ characteristic where a large change in current can occur with minimal change in electrode voltage, any increase in the applied voltage being dropped across the limiting resistor. However, the increase in current may well lead to a greater electric field asymmetry with enhanced cathode and reduced anode fields. Under such conditions, the potential difference between the plasma and anode is likely to be lower than under symmetrical field conditions, and this will be reflected in a reduction in temperature.

\section{Conclusions}

A simple dc plasma discharge represents a desirable tool to be integrated in lab-on-the-chip devices: it is simple and practical, and it offers useful emission over a wide spectrum. In this paper, an argon micro plasma discharge has been studied in order to analyse the electron energy distribution and derive an effective electron temperature. Number densities against energy are obtained from the measured emission intensity, assuming only electron-neutral collisions. By modelling the plasma using a volume averaged EEDF with a MaxwellBoltzmann distribution, an estimate of electron temperature is obtained that results in an effective temperature characteristic 
of the specific plasma parameters. The assumption of Maxwell-Boltzmann is reasonable when dealing with plasma emission since hot electrons are unlikely to play a significant role due to the low excitation cross-sections at high energy. The effective temperature was observed to decrease with increasing pressure as expected. The decrease in temperature with increasing current, for an almost constant electrode voltage, suggests a reduction in plasma-anode potential difference as the electric field distribution becomes asymmetrically enhanced at the cathode.

\section{References}

[1] Mariotti D, Shannon J, Schreitmueller H and McLaughlin J 2000 Proc. Int. Conf. on Gas Discharges and their Applications (Glasgow) vol 2, p 760

[2] Bilgic A M, Engel U, Voges E, Kuckelheim M and Broekaert J A C 2000 Plasma Sources Sci. Technol. 91

[3] Engel U, Bilgic A M, Haase O, Voges E and Broekaert J A C 2000 Anal. Chem. 72193

[4] Eijkel J C T, Stoeri H and Manz A 2000 Anal. Chem. 722547

[5] Iza F and Hopwood J 2002 Plasma Sources Sci. Technol. 11229

[6] Miclea M, Kunze K, Franzke J and Niemax K 2002 Spectrochim. Acta B 571585

[7] Dubitsky W, Mariotti D, Hyland M, McLaughlin J A and Maguire P 1999 Int. J. Comput. Appl. 688

[8] Hyland M, Mariotti D, Dubitzky W, McLaughlin J A and Maguire P 2000 Proc. SPIE Int. Symp. on Optical Science vol 4120, p 246

[9] Lemoine P, Mariotti D, Maguire P and McLaughlin J A 2001 Thin Solid Films 401196
[10] Gottscho R A and Miller T A 1984 Pure Appl. Chem. 56189

[11] Wilson C G, Gianchandani Y B, Arslanbekov R R, Kolobov V and Wendt A E 2003 J. Appl. Phys. 942845

[12] Mariotti D, McLaughlin J and Maguire P 2004 Plasma Sources Sci. Technol. 13207

[13] Bogaerts A and Gijbels R 1998 J. Anal. At. Spectrosc. 13721

[14] Bogaerts A and Gijbels R 1998 J. Appl. Phys. 84121

[15] Exciatation cross sections, available from: http://jilawww. colorado.edu/www/research/colldata.html

[16] National Institute of Technology, NIST Atomic Spectra Database, NIST Standard Reference Database \#78. Available from: http://www.nist.gov

[17] Lilly R A 1976 J. Opt. Soc. Am. 66245

[18] Bogaerts A, Quentmeier A, Jakubowski N and Gijbels R 1995 Spectrochim. Acta $\mathbf{5 0} 1337$

[19] Savukov I M 2003 J. Phys. B: At. Mol. Opt. Phys. 362001

[20] Jelenak Z M, Velikic Z B, Bozin J V, Petrovic Z Lj and Jelenkovic B M 1993 Phys. Rev. E 473566

[21] Lieberman M A and Licthenberg A J 1994 Principles of Plasma Discharges and Material Processing (New York: Wiley-Interscience)

[22] Schutze A, Jeong J Y, Babayan S E, Park J, Selwyn G S and Hicks R F 1998 IEEE Trans. Plasma Sci. 261685

[23] Behringer K and Fantz U 1994 J. Phys. D: Appl. Phys. 272128

[24] Meijer F G 1996 Trans. Fusion Technol. 29342

[25] Bogaerts A 1999 Plasma Sources Sci. Technol. 8210

[26] Braithwaite N St J 2000 Plasma Sources Sci. Technol. 9517

[27] Puech V and Torchin L 1986 J. Phys. D: Appl. Phys. 192309

[28] Haas F A and Braithwaite N St J 2000 Plasma Sources Sci. Technol. 977

[29] Bogaerts A, Gijbels R and Vlcek J 1998 Spectrochim. Acta 53 1517

[30] Yang C and Harrison W W 2001 Spectrochim. Acta 561195 